\title{
Klinikreform
}

\section{Grenze zwischen ambulant und stationär wird durchlässig}

\author{
Nicht nur über die Portalpraxen wird die Krankenhausstrukturreform \\ auf den ambulanten Sektor ausstrahlen. Praxisinhaber sollten auch im \\ Blick behalten, wie sich der geplante Strukturfonds auf das ambulante \\ Versorgungsangebot niederschlagen wird.
}

$\mathrm{D}$ ie Bestimmungen des Krankenhausstrukturgesetzes werden in den kommenden Jahren auch in den ambulanten Sektor hineinwirken, niedergelassene Ärzte sollten sich darauf einstellen. Das gilt nicht nur für die viel diskutierten Portalpraxen, sondern auch für den Strukturfonds oder weitere Detailregelungen zur Qualitätssicherung. Diese These hat Georg Heßbrügge, Bereichsleiter Gesundheitsmärkte und -politik bei der Deutschen Apotheker- und Ärztebank (apoBank), im Gespräch mit Springer Medizin vertreten. Mit dem Strukturfonds will der Bund bekanntlich einen Beitrag dazu leisten, die Zahl der Kliniken zu reduzieren. Er stellt dafür 500 Millionen Euro bereit, die Länder sollen ebenso viel Geld beisteuern. Die Fördermittel sollen dafür verwendet werden, dass Kliniken geschlossen werden - und dann zum Beispiel in Gesundheitszentren, etwa für die geriatrische Versorgung, oder in Pflegeeinrichtungen umgewidmet werden.

\section{Neue Kooperationen sind möglich}

„Niedergelassene Ärzte sollten auf jeden Fall in ihrer Region die Augen offen halten, was sich bei den Krankenhäusern im Einzelnen tut", empfiehlt Heßbrügge. Durch die Umwidmung eines Krankenhauses etwa in ein geriatrisches Zentrum könnte sich die Konkurrenzsituation verändern - im negativen wie auch im positiven Sinne. Es kommt auf den konkreten Einzelfall an. Fest steht, die Versorgungslandschaft verändert sich, und die Beteiligten müssen darauf re- agieren. Das könnte beispielsweise eine Kooperation mit dem neu entstehenden Pflegeheim sein oder ein Gesundheitszentrum. Ebenso können aber auch Maßnahmen nötig werden, die eine Arztpraxis vor Ort stärken. Es sei vor allem abzuwarten, welcher Landrat tatsächlich bereit sei, auf „seine“ Klinik zu verzichten, denn das Antragsrecht auf "Umwidmung" liegt bei den Ländern im Einvernehmen mit den Landesverbänden der Krankenkassen. Immerhin könnten neu entstehende ambulante Gesundheitszentren helfen, Versorgungslücken, die in den kommenden Jahren erwartet werden, teilweise zu schließen.

In den Städten gebe es dagegen keine Probleme, die Sicherstellung zu gewährleisten. Das gelte im Prinzip auch für die Notfallversorgung. Dafür haben die KVen nach wie vor den Sicherstellungsauftrag, was noch einmal durch die "Soll-Regelung" deutlich wird. Ausreichend gesetzliche Freiräume seien wichtig, um den unterschiedlichen regionalen Gegebenheiten gerecht zu werden, so Heßbrügge. In manchen Städten gibt es bereits Beispiele für an den Kliniken angesiedelte und gut funktionierende Notfalldienste, die von KVen organisiert werden, wie etwa in Dortmund.

\section{Innovationsbereitschaft gefragt}

Letztlich reihen sich die Regelungen der Krankenhausstrukturreform in viele weitere Gesetzesänderungen ein, die in den letzten Jahren auf den Weg gebracht wurden. Von der Öffnung der Universitätsambulanzen, über die Ermächtigung

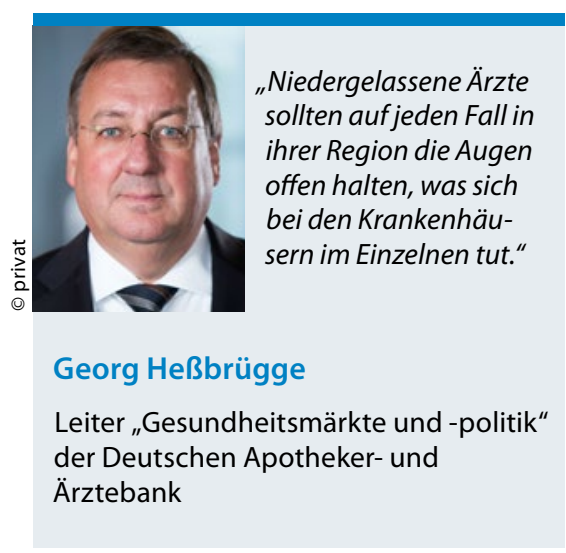

der Krankenhäuser bei Unterversorgung bis hin zum Strukturfonds: „Der Gesetzgeber erleichtert es der stationären Seite, in die ambulante Versorgung hineinzugehen, und weicht die Grenze zwischen ambulant und stationär immer weiter auf." Am Ende stehe die Frage: „Wer macht ambulante Medizin und kriegt auch Geld dafür?" Das Zusammenführen der Sektoren muss aber für beide Seiten je nach den jeweiligen Gegebenheiten auch in Zukunft lebbar sein und nicht zu einem Verdrängungswettbewerb führen.

Vieles sei in der Reform „richtig adressiert", resümiert Heßbrügge, aber zu langatmig angelegt und letztlich inkonsequent. So sei es auch mit den Qualitätszu- und -abschlägen. Zunächst müsse konkretisiert werden, was Qualität überhaupt sei und wie sie zu vergüten sei. Bis die Kriterien für Qualität gefunden sind, werde noch einige Zeit ins Land gehen, vermutet er. Wenn der Kliniksektor aber einmal so weit sei, so Heßbrügge, „dann ist das auch ein Einfallstor für Pay for Performance in den Praxen".

Eine Sonderproduktion von Springer Medizin und der Deutschen Apotheker- und Ärztebank; Koordination und Redaktion: Hauke Gerlof, Neu-Isenburg 